\title{
Dengue and Chikungunya Fever: Oral Manifestations
}

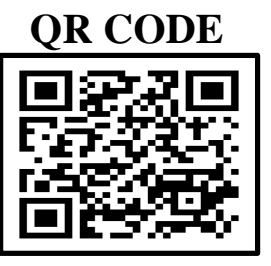

\section{INDRAJEET SINGH ${ }^{*_{1}}$, NARESH SHARMA² $^{2}$}

The vector borne diseases of dengue and chikungunya mainly affect the African and South Asian countries. Its prevalence increases in the summer and monsoon seasons due to an increase in breeding sites and External Incubation Period (EIP) of the virus. Primarily caused by an infectious bite of the Aedes aegypti female mosquito, this disease has a variety of systemic signs and symptoms. However, the oral manifesatations might be ignored. This review aims to enlightens the healthcare researchers with knowledge regarding the oral manifestations of dengue and chikungunya.

KEYWORDS: Dengue, Chikungunya, Oral, Manifestations, Ulcer, Gingival Bleeding.

\section{INTRODUCTION}

Having being ignored for many years, the incidence of dengue and chikungunya has increased over the years so much so that the disease has become an international public health concern. ${ }^{1}$ This can be seen from the fact that while only 9 countries experienced dengue epidemics before the year 1970, dengue in particular is now an endemic in about 100 countries. $^{2}$ The fact increasing the incidence of disease that dengue and chikungunya is that affected countries face multiple public health problems and hence, are unable to completely focus their attention to one specific disease due to a number of constraints (finances, manpower, resources, etc).

In addition to that, the viral diseases of dengue and chikungunya are affected by various Dengue and chikungunya are driven by complex interactions among host, vector and virus that are influenced by climatic factors. It has been reported that environmental factors like temperature, precipitation and rainfall can affect the External Incubation Period(EIP) of the virus and increase the incidence of vector borne diseases. ${ }^{3}$

India receives approximately $75 \%$ of its total rainfall during June to September(southwest monsoon period). This rainfall provides ample breeding habitats for Ae. Aegypti (causative agent for the disease), which leads to high vector densities which are difficult to control. ${ }^{4}$ This review paper provides information about the etiogenesis, systemic factors and oral manifestations of dengue and chikungunya.

\section{ETIOPATHOGENESIS OF DENGUE AND CHIKUNGUNYA}

Dengue: This virus is transmitted in humans by female Aedes (Ae.) mosquitoes which belongs to the subgenus Stegomyia. It has been reported that Ae. aegypti has been the most important epidemic vector in the tropical and subtropical regions for this disease. Other species such as Ae. albopictus, Ae. polynesiensis, member of Ae. scutellaris complex along with and Ae. niveus have been documented to play a role as secondary vectors for dengue. Studies have reported that Ae. niveus is considered only as a sylvatic vector. ${ }^{5}$

Chikungunya: This viral disease of the genus Alphavirus is transmitted to humans through infected mosquitoes (Primarily Aedes aegypti and Aedes albopictus). ${ }^{6}$ This virus was first recognized as a human pathogen during the 1950 os in Africa, following which cases have been reported in many countries in the African and Asian continent. ${ }^{7}$

\section{CLINICAL SYMPTOMS OF DENGUE AND CHIKUNGUNYA}

Dengue: As per the World Health Organization (WHO) 2011 case definition, dengue is suspected in a person with high fever and two of the following signs/symptoms:

- Headache

- Retro-orbital pain

- Myalgia

- Arthralgia/ bone pain 
- Rash

- Bleeding manifestations: petechiae, epistaxis, gum bleeding, hematemesis, melena, or positive tourniquet test.

- Leukopenia $\left(\mathrm{WBC} \leq 5,000\right.$ cells $\left./ \mathrm{mm}^{3}\right)$

- Platelet count $\leq 150,000$ cell $/ \mathrm{mm}^{3}$

- Hematocrit (Hct) rising 5-10\%. ${ }^{8}$

Chikungunya: Symptoms start abruptly with fever (temperature usually 138.9 degree Celsius). The fever typically lasts from a few days up to 2 weeks and its nature can be biphasic. After the onset of fever, a majority of infected persons develop severe along with often debilitating polyarthralgias. The joint pain is usually symmetric in nature and mostly occurs in wrists, elbows, fingers, knees, and ankles with the ability to affect more-proximal joints. Arthritis with joint swelling can also occur. Another symptom is rash, which is variable between patients. ${ }^{10}$

\section{ORAL MANIFESTATIONS OF DENGUE AND CHIKUNGUNYA}

Dengue: Various authors have the documented the following oral manifestations of dengue. The list is exhaustive, but not limited to:

1. Rashes

2. Petechiae

3. Bleeding gums

4. Ulcers

5. Dryness of mouth

6. Lips encrusting11

7. Small vesicles on the soft palate ${ }^{12}$

8. Erythema of lips ${ }^{12}$

9. Hemorrhagic plaques on both buccal mucosa ${ }^{13}$

10. Enlarged and inflamed tonsils ${ }^{14}$

11. Xerostomia ${ }^{14}$

Chikungunya: The clinical manifestations are as follows:

1. Morbilliform eruption ${ }^{15}$

2. Rash $^{16}$

3. Mild pruritus ${ }^{16}$

4. Aphthous ulceration ${ }^{17,18}$

5. Depigmented macules on lips

6. Crusted lesions on the lips and angle of mouth

7. Oral mucosal pigmentation

8. Depigmented macules on lips

9. Crusted lesions on the lips and angle of mouth

10. Oral mucosal pigmentation ${ }^{15}$

11. Multiple aphthae, erosions and cheilitis ${ }^{14}$
12. Pain ${ }^{14}$

13. Mucopyrosis

14. Bleeding from the gingiva

15. Difficulty in chewing and swallowing

16. Halitosis

17. Trismus

18. Excessive salivation and distaste

19. Mobile teeth ${ }^{19}$

\section{CONCLUSION}

In light of the above-mentioned oral manifestations of dengue and chikungunya, it is important that attention be paid to its oral manifestations so that a dentist can address those lesions, or atleast provide symptomatic relief for the same. An oral checkup of such patients can also help in providing treatment for the same.

\section{REFERENCES}

1. Kumar D, Garg S. Economic burden of dengue fever on households in Hisar district of Haryana state, India. Int J Adv Med Health Res 2014;1:99-103

2.https://www.who.int/news-room/fact-sheets/detail

/dengue-and-severe-dengue [Last Assessed on $13^{\text {th }}$ February, 2019]

3. Mutheneni SR, Morse AP, Caminade C, Upadhyayula SM. Dengue burden in India: recent trends and importance of climatic factors. Emerging Microbes \& Infections (2017) 6, e7o.

4. Angel B, Joshi V. Distribution and seasonality of vertically transmitted dengue viruses in Aedes mosquitoes in arid and semi-arid areas of Rajasthan, India. J Vector Borne Dis 2008; 45: 56-59.

5. Malavige GN, Fernando S, Fernando DJ, Seneviratne SL. Dengue viral infections. Postgraduate Medical Journal 2004;80(948):588-601.

6. https://www.who.int/denguecontrol/arbo-viral/ other arboviral chikungunya/en/ [Last Assessed on $13^{\text {th }}$ February, 2019]

7. Jupp PG, McIntosh, BM. Chikungunya virus disease. In: Monath TP, ed. The arboviruses: epidemiology and ecology vol. II. Boca Raton, FL: CRC Press, 1988:137-57.

8. WHO SEARO. Comprehensive Guidelines for Prevention and Control of Dengue and Dengue Haemorrhagic Fever Revised and expanded 2011

9. Deller JJ, Jr., Russell PK. Chikungunya disease. Am J Trop Med Hyg 1968; 17:107-11.

10. Staples JE, Breiman RF, Powers AM. Chikungunya Fever: An Epidemiological Review of a Re-Emerging 
Infectious Disease. Clinical Infectious Diseases 2009; 49:942-8.

11. Govindaraj S, Jayaraman R, Daniel M J, Subbiah S, Vasudevan SS, Kumaran JV. Oral manifestations of dengue fever. Sahel Med J 2018;21:194-8

12. Chadwick D, Arch B, Wilder-Smith A, Paton N. Distinguishing dengue fever from other infections on the basis of simple clinical and laboratory features: 2006;35:147-53.

13. Thomas EA, John M, Kanish B. Mucocutaneous manifestations of dengue fever. Indian J Dermatol.2010;55:79-85.

14. Mobeen N. Oral Manifestations of dengue and chikungunya fever. J Pharm Sci \& Res 2015;7(9):76971.

Source of support: Nil, Conflict of interest: None

\section{$\frac{\text { AUTHOR AFFILIATIONS: }}{1 .}$}

1. BDS, Private Practitioner, Greater Noida

2. BDS, Private Practitioner, Bulandshehr
15. Bandyopadhyay D, Ghosh SK. Mucocutaneous Manifestations of Chikungunya Fever. Indian J Dermatol. 2010; 55(1): 64-7.

16. Bandyopadhyay D, Ghosh SK. Mucocutaneous features of Chikungunya fever: a study from an outbreak in West Bengal, India. Int J Dermatol. 2008;47(11):1148-52.

17. Bhat RM, Rai Y, Ramesh A, Nandakishore B, Sukumar D, Martis J, Kamath GH. Mucocutaneous Manifestations of Chikungunya Fever: A Study From An Epidemic In Coastal Karnataka. Indian J Dermatol. 2011; 56(3):290-4.

18. Katti R, Shahapur P R, Udapudi K L. Impact of chikungunya virus infection on oral health status: An observational study. Indian J Dent Res 2011;22:613. 